\title{
Silicon Micromachined Electromagnetic Microactuators for Rigid Disk Drives
}

\author{
Weilong Tang ${ }^{1}$, Viktoria Temesvary ${ }^{1}$, Raanan Miller ${ }^{2}$, Amish Desai ${ }^{2}$, \\ Yu-Chong Tai ${ }^{2}$ and Denny K. Miu ${ }^{1}$ \\ ${ }^{1}$ Mechanical Engineering, UCLA, Los Angeles, CA 90024-1597 \\ 2 Electrical Engineering, Caltech, 116-81, Pasadena, CA 91125
}

\begin{abstract}
It is projected that by the year 2001, disk drive industry will be shipping products with track density on the order of 25,000 tracks-per-inch, which would require a scrvo bandwidth of at least $3 \mathrm{kHz}$. This paper presents initial fabrication results of an industry and government supported research project at Caltech and UCLA to develop piggyback electromagnetically driven microactuators for such applications, which are fabricated using the state-of-the-art silicon micromachining techniques.
\end{abstract}

\section{Introduction}

Since 1991, track density of magnetic recording rigid disk drives has gone up approximately $30 \%$ every year. Together with a similar increase in bit density, they enable the industry to maintain an annual growth of areal recording density on the order of $60 \%$. Currently, the industry is shipping products with track density in excess of 5,000 tracks-per-inch (TPI) which is cxpected to increase to 12,000 by the year 1998 and to 25,000 by 2001. At a track density of 25,000 , the track-to-track spacing is $1 \mu \mathrm{m}$ and the allowable off-track error is less than $120 \mathrm{~nm}$.

There are many sources of off-track errors in rigid disk drives which include contributing factors such as mechanical resonances, spindle runouts, temperature drifts, humidity variations, external shocks/vibrations, bearing hysteresis, cable bias, servo pattern errors, and residual vibrations, etc. Obviously, the higher the servo bandwdith, the easier it is to correct these errors; and the higher the track density, the more important it is to have high-bandwidth servo system. Unfortunately, servo bandwidth of computer rigid disk drives for the last ten years has been limited to less than $600 \mathrm{~Hz}$, mainly by unavoidable inherent in-the-loop mechanical resonances. For 25,000 TPI, it is projected that servo bandwidth must be higher than $3 \mathrm{kHz}$; furthermore, with spindle speed increased to 7,200 RPM, the required bandwidth will increase to $4 \mathrm{kHz}$.
It is well recognized throughout the industry that this level of performance simply cannot be achieved by using or improving upon the existing hardware. Ono solution is to add a dual-stage piggyback microactuator system similar to that of optical drives (see References [1] and [2]).

At UCLA and Caltech, for the last three years, we have been involved in a collaborative effort to develop micromachined electromagnetcially driven microactuators for future high-track-density computer disk drives.

\section{Results and Discussions}

Figure 1 shows a previously documented silicon microgimbal which is now used as the structural platform for a planar electromagnetically driven piggyback microactuator (see Reference [3]). This active device is fabricated within the center coupon of the silicon microgimbal such that the $\mathrm{R} / \mathrm{W}$ slider can be moved relative to the gimbal/suspension assembly. This approach has the advantage that any structural resonances in the suspension arm is completely eliminated from the servo loop, allowing a substantial increase in servo bandwidth. In addition, this design can be very low-profile such that it is consistent with the disk-to-disk spacing requirement of future products.

Figure 2 shows a detail schematic of the planar variable reluctance microactuator which uses wrap-around copper coils (the entire device fits inside the center coupon of the microgimbal which measures less than 1.5 $\mathrm{mm}$ square). Instcad of a solid platform, a. sct of hairpin-like planar microsprings are micromachined into the silicon microgimbal to allow in-plane motion relative to the outside frame. Unlike the serpentine springs of the microgimbal, these planar microsprings are designed to have maximum in-plane compliance while maintaining adequate out-of-plane, pitch and roll stiffnesses.

As shown in Figure 2, a pair of thin-film variablereluctance microactuators are used to move the slider/recording head in the in-plane direction, which is attached to the center movable permalloy core. The two stationary enclosing permalloy cores of the microac- 
tuator are mounted on the outside frame of the center coupon of the microgimbal and are constructed of one layer of permalloy with wrapped-around helical flat copper coils. When a voltage is applied, magnetic force will be exerted on the slider, pulling it towards the stator and resulting in in-plane/cross-track micron-level fine motion. This design has the important advantage that the required fabrication technology is very similar to that of thin-film inductive heads, leveraging the expertise of the magnetic recording industry.

Figure 3 summaries the fabrication process for the electromagnetic microactuator with wrap-around copper coils which requires six masks, in addition to those necessary for the microgimbals. After oxidation and back-etch, the first step involves thermal evaporation of the titanium/copper seed layer and patterning of the photoresist electroplating mold for the bottom copper traces. Subsequent to electroplating and removal of the seed layer, a thin layer of photoresist will be patterned and hot baked to form an insulation layer. Then another seed layer will be evaporated and permalloy will be electroplated using the frame-plating technique typical of the thin-film head industry.

Finally another photoresist insulation layer and the top copper will be deposited which wraps around the permalloy core and makes contact with the bottom copper layer to form complete copper coils. At the end of the microactuator fabrication, thick photoresist is patterned as the RIE mask and both the serpentine microsprings of the microgimbal and the hair-pin planar microsprings of the microactuators will be plasma etched until free-standing. Figure 4 shows one of our first working prototypes.

The advantage of the microactuator design as shown in Figures 2 and 4 is that it is very space efficient such that a large number of turns can be packaged in a given real estate, maximizing the available reluctance force. The disadvantages, however, are many. Since there are many contact vias between the two copper layers, as is well known in the thin-film head industry, the electrical resistance could be quite high and more importantly, production yield could be a potential problem. A better design would be that of a planar copper coil similar to that of the thin-film head.

Furthermore, since the in-plane dimensions are rather limited due to the size of the microgimbal (which has to be consistent with that of the $30 \%$ form factor pico-sliders), the only way to increase force, in addition to increasing the number of turns, is to increase the thickness of the permalloy cores. Specifically, for the variable-reluctance microactuator, the resulting force is

$$
F=0.5 \mu_{0} N^{2} I^{2} w t g^{-2}
$$

where $N=$ number of turns, $I=$ current, $w=$ width, $t=$ thickness of permalloy and $g=$ gap. If we assume that in the worst case the microactuator has to move a pico-slider (with mass $M=2 \mathrm{mg}$ ) at a displacement of $\delta=0.5 \mu \mathrm{m}$ (since the current VCM design is capable of $5,000 \mathrm{TPI}$ ) at $\omega=3 \mathrm{kHz}$, the force required is

$$
F=M \delta \omega^{2}=0.3 \mathrm{mN}
$$

then for $N=40, I=85 \mathrm{~mA}, w=.25 \mathrm{~mm}, g=10$ $\mu \mathrm{m}$, the required permalloy thickness $t$ is $20 \mu \mathrm{m}$. In the current design, increasing the permalloy thickness is a potential problem for the step coverage of the top copper layer.

Finally and most importantly, as can be seen from Figures 2 and 4, the contact area between the movable platform and the slider is very small, potentially degrading the mechanical integrity of the entire system. Figure 5 shows an alternative design where instead of a copper coil wrapped around a single layer of permalloy, two layers of planar coils are sandwiched between two layers of permalloy. In this design, the top half of the center coupon is devoted to the variable reluctance microactuators and the bottom half to the planar microsprings, significantly increases the contact area between the movable platform and the slider.

The fabrication process for the electromagnetic microactuator with planar coils requires four additional masks compared to the earlier design. In this new design, cavities are first plasma-etched into the silicon substrate and the bottom permalloy layer is electroplated into the cavities which are subsequently planarized using photoresist insulation layer. After the deposition of two layers of planar copper coils and insulations, the top layer of permalloy is electroplated which makes contact with the bottom layer, completing the magnetic circuit. Since the permalloy layers are either buried into the silicon or is fabricated in the last photolithography step before RIE, step coverage is less of a problem resulting in a thicker permalloy (at least $10 \mu \mathrm{m}$ ). Figure 6 shows a fabricated electromagnetic microactuator with planar copper coils.

More results related to design, analysis and testing of these micromachined devices will be presented in a later paper (see [4]).

\section{References}

1] Mori, K., ..., "A Dual-Stage Magnetic Disk Drive Actuator Using A Piezoelectric Device For A High Track Density," IEEE Trans. on Magn., Vol. 27(6), 1991, pp. 5298 5300.

[2] Fan, L.-S., ..., "Magnetic Recording Head Positioning at Very High Track Densities Using a Microactuator-Based, Two-Stage Servo System," to appear in the IEEE Trans. on Indust. Elect., 1995.

[3] Temesvary, V., ..., "Design, Fabrication and Testing of Silicon Microgimbals for Super-Compact Rigid Disk Drives," IEEE/ASME J. of MEMS, Vol. 4, No. 1, March 1995, pp. 18-27.

[4] Tang, W., ..., "Design, Fabrication and Testing of Micromachined Electromagnetic Microactuators for Rigid Disk Drives," in preparation. 


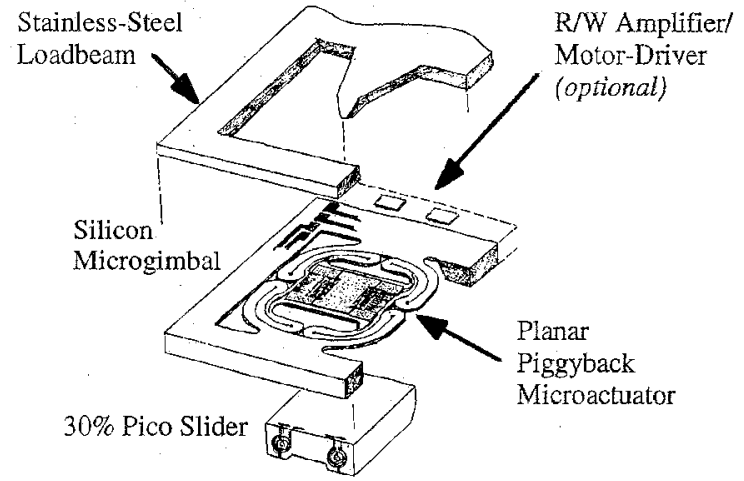

Figure 1: Design concept for a silicon micromachined electromagnetic piggyback planar microactuator for high TPI applications.

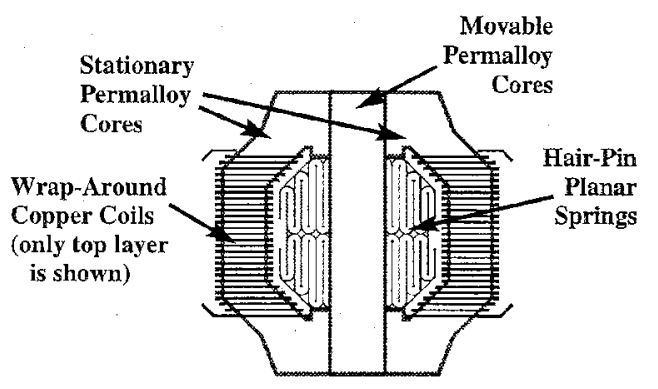

Figure 2: Schematic drawing of the silicon microactuators with wrap-around copper coils.

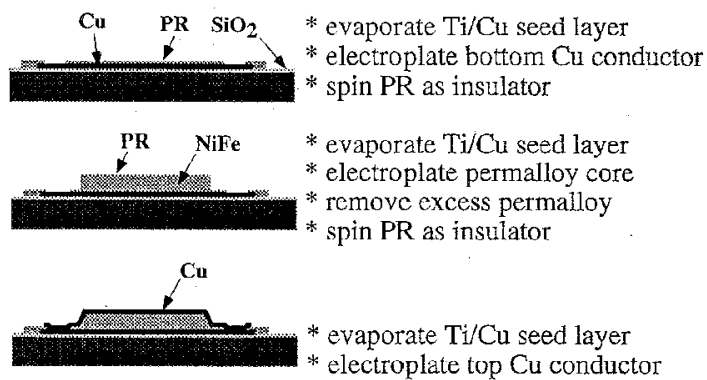

Figure 3: Processing steps for the silicon microactuators with wrap-around copper coils.

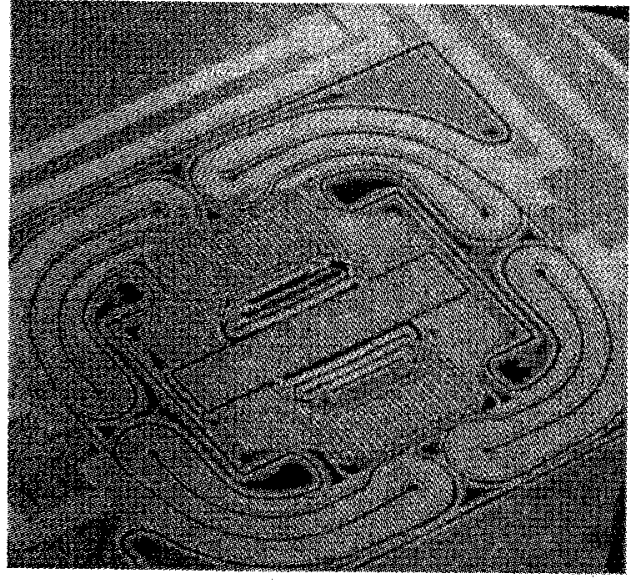

Figure 4: SEM micrograph of a fabricated electromagnetic microactuator with wrap-around copper coils (the length of the center coupon is about $1.25 \mathrm{~mm})$.

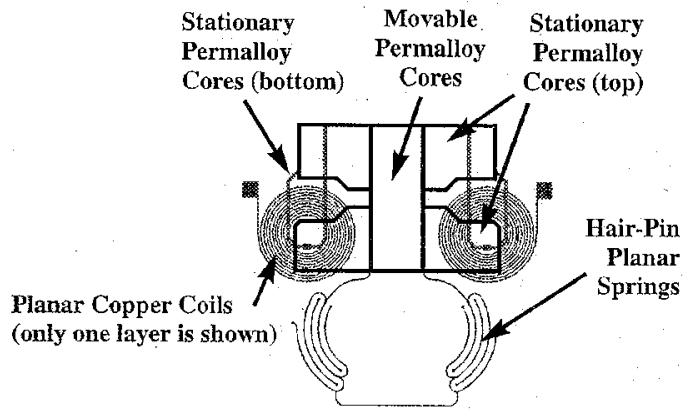

Figure 5: Schematic drawing of the silicon microactuators with planar copper coils.

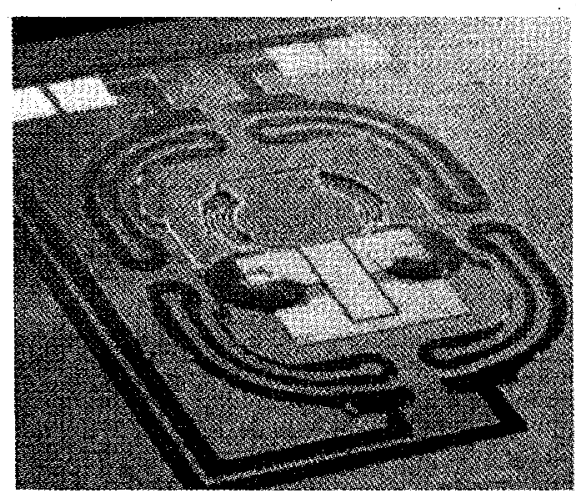

Figure 6: SEM micrograph of a fabricated electromagnetic microactuator with planar copper coils. 